(2) Open Access Full Text Article

\title{
Accuracy of B-scan ultrasonography in acute fundus obscuring vitreous hemorrhage using a standardized scanning protocol and a dedicated ophthalmic ultrasonographer
}

\author{
This article was published in the following Dove Press journal: \\ Clinical Ophthalmology \\ 27 July 2017 \\ Number of times this article has been viewed
}

\section{Maria T Sandinha' \\ Ajay K Kotagiri' \\ Rona I Owen' \\ Caspar Geenen' \\ David HW Steel ${ }^{1,2}$}

'Sunderland Eye Infirmary, Sunderland, ${ }^{2}$ nstitute of Genetic Medicine, Newcastle University, Newcastle upon Tyne, UK
Correspondence: Caspar Geenen Sunderland Eye Infirmary, Queen Alexandra Road, Sunderland, Tyne and Wear, SR2 9HP, UK

Tel +44 I9I 5656256

Fax +44 I9I 5140220

Email caspargeenen@gmail.com
Purpose: To assess the accuracy of B-scan ultrasound $(\mathrm{U} / \mathrm{S})$ in diagnosing cases of acute fundus obscuring vitreous hemorrhage $(\mathrm{FOVH})$ using a standardized scan protocol and dedicated ophthalmic ultrasonographer.

Methods: Consecutive patients presenting with acute FOVH of unknown cause, between January 2013 and December 2014, were prospectively recruited. Patients underwent a scan performed by a dedicated ultrasonographer, utilizing a systematic scan sequence and using an ocular specific U/S device. The U/S findings were compared to the findings during vitrectomy or after spontaneous hemorrhage clearance.

Results: Fifty-eight eyes (58 patients) were included. An underlying rhegmatogenous retinal detachment (RRD) and retinal tears without RRD were reported in nine and 14 patients, respectively. Nineteen of these patients underwent vitrectomy, and the other four underwent laser retinopexy or cryopexy alone. An additional six patients with suspected but uncertain retinal tears underwent vitrectomy, during which tears were confirmed in three, two had retinal vessel avulsions, and one had retinal new vessels. There was "complete" agreement between the B-scan findings and clinical findings in $78 \%$ of patients, "partial" agreement in 19\%, and agreement was not tested in 3\%. When the agreement was "partial", the disagreements did not affect patient management. The sensitivity was $100 \%$ for the detection of RRD, and for the detection of new retinal tears in patients without retinal detachment.

Conclusion: B-scan U/S scan was highly sensitive in identifying the pathology in acute FOVH. Our results show an improvement from previously reported results, likely related to the standardized scan protocol and dedicated ophthalmic ultrasonographer.

Keywords: diagnosis, ultrasonography, ultrasound B-scan, rhegmatogenous retinal detachment, retinal tear, retinal break

\section{Introduction}

The incidence of spontaneous vitreous hemorrhage is $\sim 7$ cases per 100,000 . Proliferative diabetic retinopathy (PDR; 32\%), retinal tear $(30 \%)$, proliferative retinopathy after retinal vein occlusion (RVO; 11\%), and posterior vitreous detachment (PVD) without a retinal tear $(8 \%)$ are the most common causes of spontaneous fundus obscuring vitreous hemorrhage (FOVH). Vitreous hemorrhage in the setting of acute PVD has been estimated to be associated with retinal tear or a break in $70 \%-95 \%$. ${ }^{1}$

It is important to identify an underlying retinal tear or rhegmatogenous retinal detachment (RRD) urgently in these cases, as this significantly changes the management 
plan. Conversely, it is also important to avoid potentially unnecessary surgery in other less urgent conditions, in which it is safe to wait and see if spontaneous hemorrhage clearance occurs. Although the medical and ocular history (eg, known PDR) may suggest the underlying cause of FOVH, B-scan ultrasound (U/S) is the only investigation available currently to assess the integrity of the posterior segment. Recent publications have recommended early pars plana vitrectomy (PPV) in cases with FOVH presumed to be resulting from retinal tears and detachments, irrespective of the U/S findings ${ }^{2,3}$ relating to the limited sensitivity of ultrasonography in detecting retinal breaks.,

Our standard practice has been for all U/S examinations on patients with FOVH to be carried out by a dedicated ophthalmic ultrasonographer using a standardized scan protocol with a purpose built ocular specific high resolution $\mathrm{U} / \mathrm{S}$ scanner, contrary to previous reports. ${ }^{2-4}$ We hypothesized that this would improve the accuracy of the U/S in detecting retinal tears and detachment. We carried out a prospective study to assess the accuracy of our U/S protocol in identifying significant underlying pathology, and we assessed the level of agreement between the U/S findings and either the perioperative findings or the clinical findings after hemorrhage resolution, depending on the management of that particular patient (ie, surgical vs observational).

\section{Methods}

The study was conducted at Sunderland Eye Infirmary, a large specialized eye hospital in Northern England. Data from consecutive patients presenting with acute FOVH and undergoing U/S from January 2013 to December 2014 were prospectively collected, using a standardized recording protocol. Patients with known PDR and RVOs were excluded. The severity of the vitreous hemorrhage was graded by ophthalmoscopy as follows: 1 ) grade 0 - significant visibility of the fundus details; 2 ) grade $1->50 \%$ of the retina visible; 3 ) grade 2 - only peripheral retinal details visible; 4) grade 3 - no fundal view possible.

Only patients with grade 1 FOVH and above and those who presented with a symptomatic history of $<1$ week were included. All patients underwent an urgent U/S B-scan (Figure 1) performed by an ultrasonographer with 20 years of experience in ocular U/S imaging, using an ophthalmic specific digital U/S scanner (Eye Cubed V3 ultrasound imaging system; Ellex Innovative Imaging, Sacramento, CA, USA). A posterior segment focused, $10 \mathrm{MHz}$, sealed, oil filled probe was used producing a two-dimensional, $52^{\circ}$, geometrically accurate sector image to a depth of $45 \mathrm{~mm}$. The scanning rate was 25 frames/second with a 256 shade gray scale and a resolution of $0.15 \mathrm{~mm}$ axially and $0.2 \mathrm{~mm}$ laterally.

A standardized protocol of examination was followed as per Atta $^{6}$ ensuring that all areas of the globe were assessed. In each patient, the probe was placed directly on the conjunctival surface using topical anesthesia and carbomer gel as a coupling medium. The scan protocol included transverse views of all four quadrants gradually extending from posterior to anterior, performed initially at high gain (90 dB) to assess the vitreous and then at a lower gain (65-70 dB) to assess the retina. The high sensitivity high gain setting ensured that low reflective vitreous echoes, for example, from vitreous cells and posterior hyaloid face were detected. The lower gain setting then improved resolution of the retinal image and allowed identification of smaller structures. Longitudinal scans of all meridians at lower gain were then performed in all cases to assist detection and localization of pathology.

Careful assessment was made especially looking for signs of PVD, abnormal vitreoretinal adhesion, retinal tears and their localization, retinal detachment, and any other significant pathology. Kinetic examination was included

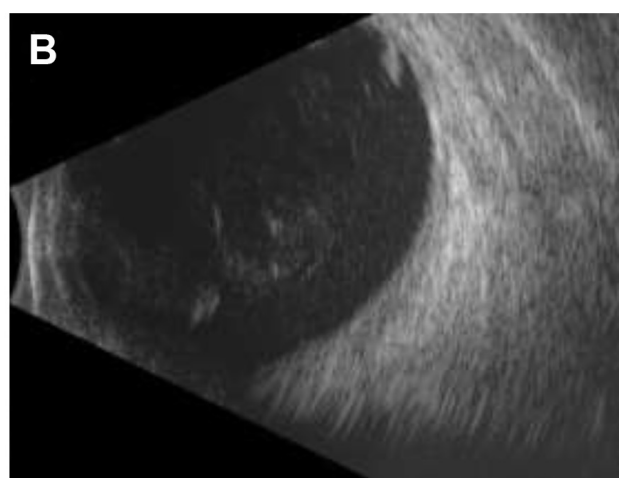

Figure I B-scan ultrasound images of a definite retinal tear.

Notes: A high gain setting of $90 \mathrm{~dB}$ was used first, revealing a posterior vitreous detachment $(\mathbf{A})$. Subsequently, the gain was lowered to $65 \mathrm{~dB}$ to assess the retina and obtain a clearer view of the tear (B). 
especially looking at areas of vitreoretinal adhesion including the vitreous base. The presence of incomplete PVD with disc attachment and/or any abnormal vitreoretinal adhesion was recorded (anteroposterior position judged primarily on the longitudinal view).

Retinal tears were graded as none, suspicious, or definite according to the judgment of the ultrasonographer. The likely diagnosis of other identified pathology was recorded, for example, retinal new vessels, submacular hemorrhage, and associated macular thickening suggestive of macular edema. Immediate (within 48 hours) PPV was performed, when an RRD was identified. It was also performed when retinal tears were identified as either definite or suspicious and laser retinopexy was not possible due to the degree of vitreous hemorrhage.

Approval from the Research Ethics Committee was not required according to national guidelines because this study was classed as a service evaluation.

The sensitivity and specificity for U/S diagnosis of RRD, and also for the presence of new non-treated retinal tears in eyes with an attached retina, were calculated with definite and suspected tears classified together. Additionally, the agreement between the U/S findings and eventual fundus findings was graded as "complete", "partial", and "none" with regard to the identified number and location of the retinal tears. If additional retinal tears were found during surgery, the agreement was graded as "partial". If retinal tears were identified perioperatively or after a period of observation, where none were identified in the U/S scan, the agreement was graded as "none". Patient demographics, presenting visual acuity, visual acuity after 3 months, final diagnosed cause of the vitreous hemorrhage, and indications for surgery were noted.

\section{Results}

During the study period, 73 patients with grade 1 FOVH and above presented and underwent ultrasonography; 15 patients were excluded due to a known previous history of PDR and RVO with laser, leaving a total of 58 patients for analysis. Patient demographics, presenting visual acuity, significant systemic and ocular history, and the grade of vitreous hemorrhage at presentation are presented in Table 1 . The majority of patients $(48 / 58)$ included in the study had either grade 2 or grade 3 vitreous hemorrhage with minimal fundus details visible. Thirty-one patients in total required PPV with 26 undergoing immediate surgery; the indications are presented in Table 2. RRD and retinal tears were the most common indications for PPV. Of the patients who did not undergo vitrectomy, none developed RRD.
Table I Study group characteristics

\begin{tabular}{ll}
\hline Total number of patients included & 58 \\
Male:female & $30(58 \%): 28(42 \%)$ \\
Age (mean, range) & $6 I(40-95)$ years \\
Right:left & $32(55 \%): 26(45 \%)$ \\
Median presenting visual acuity (range) & Counting fingers (PL - 6/9) \\
$\begin{array}{l}\text { Median visual acuity 3 months after } \\
\text { presentation (range) }\end{array}$ & $6 / 9(\mathrm{PL}-6 / 5)$ \\
Significant previous ocular history & AMD I0 \\
& Previously treated retinal \\
& tears 3 \\
& Myopia ( $>4$ diopters) 2 \\
& Previous VH of unknown \\
Systemic anticoagulants & cause I \\
Grade of vitreous hemorrhage & 4 \\
& Grade I-10 \\
Final diagnosis of cause of vitreous & Grade 2-26 \\
hemorrhage & Grade 3-22 \\
RRD & \\
Retinal tears without RRD & 9 \\
Previously treated tears with recurrent VH & 3 \\
Neovascular AMD with breakthrough VH & 6 \\
RVO & 17 \\
Trauma & 10 \\
Avulsed retinal vessel & 4 \\
PVD without tears & 3 \\
\hline
\end{tabular}

Abbreviations: AMD, age-related macular degeneration; PL, perception of light; PVD, posterior vitreous detachment; RRD, rhegmatogenous retinal detachment; $\mathrm{RVO}$, retinal vein occlusion; $\mathrm{VH}$, vitreous hemorrhage.

Table 3 provides details on the level of agreement between the U/S findings and the eventual diagnosis. There was "complete" agreement with clinical findings in 45 of the $58(78 \%)$ patients. There was "partial” agreement in $11(19 \%)$

Table 2 Management of patients with $\mathrm{FOH}$

\begin{tabular}{ll}
\hline Presumptive ultrasound diagnosis resulting in & $\begin{array}{l}\text { Number } \\
\text { of patients }\end{array}$ \\
\hline $\begin{array}{l}\text { Immediate vitrectomy } \\
\text { Retinal detachment }\end{array}$ & 9 \\
Definite retinal tears with attached retina & 10 \\
Avulsed retinal vessel with suspicion of retinal tear & 6 \\
Submacular hemorrhage due to neovascular AMD & I \\
Late vitrectomy & 3 \\
$\quad \begin{array}{l}\text { Non-resolving VH secondary to RVO } \\
\text { Previously treated retinal tears }\end{array}$ & 2 \\
Observation & 6 \\
$\begin{array}{l}\text { PVD without retinal tears } \\
\text { Retinal tears with possibility of retinopexy without } \\
\text { vitrectomy, and no other tears detected }\end{array}$ & 4 \\
$\begin{array}{l}\text { Previously treated retinal tears with recurrent VH } \\
\text { from traction }\end{array}$ & I \\
$\begin{array}{l}\text { Neovascular AMD with breakthrough hemorrhage } \\
\text { into vitreous cavity }\end{array}$ & 5 \\
RVO with NVE; one with PVD but no tears & 6 \\
Blunt trauma \\
Avulsed retinal vessel not seen on ultrasound
\end{tabular}

Abbreviations: AMD, age-related macular degeneration; $\mathrm{FOH}$, fundus obscuring hemorrhage; NVE, neovascularization elsewhere; PVD, posterior vitreous detachment; RVO, retinal vein occlusion; $\mathrm{VH}$, vitreous hemorrhage. 
Table 3 Ultrasound findings and level of agreement with eventual diagnosis, regarding the number and location of retinal tears

\begin{tabular}{|c|c|c|c|}
\hline Agreement & Ultrasound findings & Eventual diagnosis & $\begin{array}{l}\text { Number } \\
\text { of patients }\end{array}$ \\
\hline Complete & RRD & & 4 \\
\hline \multirow[t]{3}{*}{ agreement (78\%) } & Retinal tear(s) without RRD & & 14 \\
\hline & Neovascular AMD with submacular hemorrhage & & I \\
\hline & No retinal tears & & 26 \\
\hline Partial & Avulsed retinal vessel (suspicion of retinal tear) & Retinal tear & 3 \\
\hline \multirow[t]{4}{*}{ agreement (19\%) } & & Avulsed vessel without retinal tear & 2 \\
\hline & & Retinal vein occlusion with NVE & 1 \\
\hline & Retinal detachment, no retinal tear detected & RRD & 2 \\
\hline & RRD & RRD with additional retinal tear(s) & 3 \\
\hline \multirow[t]{2}{*}{ Unverified (3\%) } & & Lost to follow-up & 1 \\
\hline & & Non-clearing vitreous hemorrhage & I \\
\hline
\end{tabular}

Abbreviations: AMD, age-related macular degeneration; NVE, new vessels elsewhere; RRD, rhegmatogenous retinal detachment.

patients, although this did not affect their eventual outcome or management plan. No agreement could be verified in two patients, where one was lost to follow-up and the other had a vitreous hemorrhage secondary to a known extensive submacular hemorrhage, which did not clear and in whom surgery was not performed.

Localization of the tears in RRD was not possible in only two cases with bullous detachment of the retina. Retinal tears in an attached retina were reported in 14 cases, of which 10 underwent vitrectomy and four laser retinopexy or cryotherapy only. The four patients treated without vitrectomy were observed after treatment, with spontaneous residual hemorrhage clearance. Suspected retinal vessel avulsions with a possibility of retinal tears were reported in a further six patients and all underwent PPV, with retinal tears confirmed in three patients and avulsed vessels in two. The other case was found to have a small area of retinal neovascularization secondary to RVO. One other case was managed with immediate vitrectomy for an acute submacular hemorrhage diagnosed on U/S. Submacular hemorrhage displacement surgery with subretinal tissue plasminogen activator and intravitreal gas was carried out.

Five patients underwent late vitrectomy. Three of these patients had non-clearing vitreous hemorrhage; new blood vessels resulting from a suspected RVO were identified on the U/S scan on all three occasions. Perioperatively, these findings were confirmed and the procedure was combined with pan-retinal photocoagulation. The two other cases had recurrent vitreous hemorrhage due to previously treated tears; no new tears were found perioperatively. One other case with a previously treated tear was observed, with spontaneous hemorrhage clearance and no new tears.

Twenty-seven patients were managed conservatively; the cases are described in Table 2.
The sensitivity and specificity for the detection of retinal detachment were thus $100 \%$. In eyes with an attached retina, the sensitivity for the detection of any new retinal tears was also $100 \%$. The specificity was $90.6 \%$ with a positive predictive value of $85 \%$ and a negative predictive value of $100 \%$ (29 eyes with no new tears or RRD, three eyes with new tears falsely identified, 17 eyes with new tears identified correctly, and no eyes with new tears missed in the study period).

\section{Discussion}

The ability to detect RRD, and retinal tears in patients with an attached retina, in the presence of acute FOVH is of vital importance. These patients benefit from immediate vitrectomy if the pathology cannot be treated otherwise. We found that U/S used with the protocol described had a high sensitivity for the detection of retinal tears and the diagnosis of retinal detachment in our cohort of patients presenting with acute FOVH. None of the cases managed conservatively based on the U/S examination subsequently experienced retinal detachment and a proportion of these cleared spontaneously, avoiding vitrectomy.

Although it is standard practice to offer PPV for patients with persistent non-clearing vitreous hemorrhage, ${ }^{2,3}$ there is no consensus about the management of acute FOVH with no identifiable retinal tears and detachment on U/S. Traditionally, a conservative approach has been recommended, as there is a significant possibility of spontaneous clearance. ${ }^{7,8}$ However, recently, this management has been questioned with wide variability in the sensitivity $(44 \%-92 \%)$ of $U / S$ in the detection of retinal tears reported and the possibility of retinal detachment occurring during conservative management. ${ }^{2-5,7-10}$ Furthermore, recent technological advances in vitreoretinal surgery with lower complication rates have facilitated a trend toward early surgery. ${ }^{11}$ In our study, U/S identified all nine 
cases of retinal detachment correctly and more importantly also identified all the high-risk patients with retinal tears in an attached retina. In two cases with bullous retinal detachment on $\mathrm{U} / \mathrm{S}$, tears were not identified, presumably relating to the tear operculum lying in plane with the detached retina. There were also three instances of other patients with RRD, where additional tears were found during surgery. It is possible that these retinal tears developed after the U/S scan was performed or during the surgery. It has been stated that up to one-third of patients with PVD and acute FOVH may develop new retinal tears during follow-up, thought to be due to progressive PVD in those cases with an incomplete PVD initially. ${ }^{12,13}$ We specifically looked for evidence of incomplete PVD but did not find any cases in our cohort. In cases where it is detected, consideration should be given to repeat U/S until the PVD is complete or vitrectomy has been carried out, but an accurate and sensitive initial examination is clearly of vital importance. It is also essential that the scan is interpreted in close liaison with the surgical team managing the case, so that the significance of an incomplete PVD or vitreoretinal adhesion without a tear can be assessed in context with the likelihood of a delayed tear or detachment.

In six patients, an avulsed retinal vessel was reported with the possibility of a tear; these were graded as "partial" agreement. All underwent immediate vitrectomy and in three cases tears were found in the identified location. Caution should be exercised when avulsed vessels are reported in U/S and our findings suggest that these patients should be treated as patients with retinal tears. Previous studies have concluded that retinal tears needed to be large to be accurately diagnosed with small tears often being missed. ${ }^{9,14}$ We did not systematically grade the size of tears in this study but all were less than one clock hour in size. The ability to detect any tears or RRD is vital but the specificity in diagnosis with $\mathrm{U} / \mathrm{S}$ is also important to avoid unnecessary surgery, which has a well-known side effect profile including cataract development. Only three patients in our series underwent immediate vitrectomy for erroneously suspected retinal tears. Melamud et al recently published a comprehensive description of the clinical care of 92 patients presenting with acute FOVH. ${ }^{15}$ Thirteen eyes that underwent vitrectomy ultimately were found to have a diagnosis other than PVD as the cause of their FOVH. The rate of cataract formation after vitrectomy was high with 32 out of 49 phakic patients undergoing cataract surgery by the study end. As the authors point out, there are several factors to be considered when recommending vitrectomy in FOVH separate from the prevention or treatment of RRD, but potentially these patients could have been managed conservatively.
In comparing our series to others case mix has to be considered. We excluded patients with established proliferative disease from either diabetic retinopathy or ischemic RVO, as there was a known cause for FOVH in these patients and these may have confounded our results. We also clinically graded the degree of vitreous hemorrhage to allow further interpretation of our data. Previous studies have not done this; so it is difficult to directly compare our results with theirs. However, our series clearly had a very high sensitivity and specificity for the detection of RRD and retinal tears in comparison to other recent series. ${ }^{4,5,9}$ Lorenzo-Carrero et al also reported a high sensitivity and specificity when using U/S to screen for retinal tears in acute symptomatic PVDs without FOVH; ${ }^{12}$ like us they also used a standardized protocol. There have also been significant advances in the technology of U/S scans used in ophthalmology. Many previous studies estimating the sensitivity of the U/S in identifying retinal tears were performed using older and non-ophthalmic-specific U/S machines. Significantly, all the scans were performed by one proficient ultrasonographer, who was also an experienced ophthalmologist able to interpret the significance of subtle signs in reaching a diagnosis. We believe this is also a very important factor in our results. As in any skilled task, experience will improve performance. Training and limiting the performance of U/S to designated personnel are key factors to be considered in all ophthalmic units.

This study has several limitations. Data were collected over a 2-year period and a larger cohort would add greater validity to our findings. Similarly, the study was also single center and duplication of the effect of a single experienced ultrasonographer using a standardized scan protocol with an ophthalmic specific U/S is needed. In two patients, we could not validate the U/S findings - one defaulted from follow-up after 2 months and the other had a hemorrhage that did not clear. In both cases, however, there was no RRD at last follow-up.

\section{Conclusion}

We found that the sensitivity and specificity of U/S in the detection of retinal tears and RRD in patients with acute FOVH were higher than previously reported. This could be related to the use of a standardized scan protocol, an ophthalmic specific U/S device, and one experienced ultrasonographer performing all scans.

\section{Disclosure}

The authors report no conflicts of interest in this work. 


\section{References}

1. Spraul CW, Grossnoklaus HE. Vitreous hemorrhage. Surv Ophthalmol. 1997;42:3-39.

2. Tan HS, Mura M, Bij1 HM. Early vitrectomy for vitreous hemorrhage associated with retinal tears. Am J Ophthalmol. 2010;150:529-533.

3. Dhingra N, Pearce I, Wong D. Early vitrectomy for fundus-obscuring dense vitreous hemorrhage from presumptive retinal tears. Graefes Arch Clin Exp Ophthalmol. 2007;245:301-304.

4. Rabinowitz R, Yagev R, Shoham A, Lifshitz T. Comparison between clinical and ultrasound findings in patients with vitreous hemorrhage. Eye. 2004;18:253-256.

5. DiBernardo C, Blodi B, Byrne SF. Echographic evaluation of retinal tears in patients with spontaneous vitreous hemorrhage. Arch Ophthalmol. 1992; 110:511-514.

6. Atta HR. Ophthalmic Ultrasound-A Practical Guide. London: Churchill Livingstone; 1996.

7. Lincoff H, Kreissig I, Wolkstein M. Acute vitreous hemorrhage: a clinical report. Br J Ophthalmol. 1976;60:454-458.

8. Wilkinson CP. What ever happened to bilateral patching? Retina. 2005; 25:393-394.
9. Nischal KK, James JN, McAllister J. The use of dynamic ultrasound $\mathrm{B}$-scan to detect retinal tears in spontaneous vitreous hemorrhage. Eye. 1995;9:502-506.

10. Sarrafizadeh R, Hassan TS, Ruby AJ, et al. Incidence of retinal detachment and visual outcome in eyes presenting with posterior vitreous separation and dense fundus obscuring vitreous hemorrhage. Ophthalmology. 2001;108:2273-2278.

11. Gupta B, Sivaprasad S, Wong R, et al. Visual and anatomical outcomes following vitrectomy for complications of diabetic retinopathy: the Drive UK study. Eye. 2012;26:510-516.

12. Lorenzo-Carrero J, Perez-Flores I, Cid-Galano M, et al. B-scan ultrasonography to screen for retinal tears in acute symptomatic age-related posterior vitreous detachment. Ophthalmology. 2009;116:94-99.

13. Carrero JL. Incomplete posterior vitreous detachment: prevalence and clinical relevance. Am J Ophthalmol. 2012;153:497-503.

14. Lindgren G, Sjodell L, Lindblom B. A prospective study of dense spontaneous vitreous hemorrhage. Am J Ophthalmol. 1995;119:458-465.

15. Melamud A, Pham H, Stoumbos Z. Early vitrectomy for spontaneous, fundus-obscuring vitreous hemorrhage. Am J Ophthalmol. 2015;160: 1073-1077.
Clinical Ophthalmology

\section{Publish your work in this journal}

Clinical Ophthalmology is an international, peer-reviewed journal covering all subspecialties within ophthalmology. Key topics include: Optometry; Visual science; Pharmacology and drug therapy in eye diseases; Basic Sciences; Primary and Secondary eye care; Patient Safety and Quality of Care Improvements. This journal is indexed on

\footnotetext{
Submit your manuscript here: http://www.dovepress.com/clinical-ophthalmology-journal
}

\section{Dovepress}

PubMed Central and CAS, and is the official journal of The Society of Clinical Ophthalmology (SCO). The manuscript management system is completely online and includes a very quick and fair peer-review system, which is all easy to use. Visit http://www.dovepress.com/ testimonials.php to read real quotes from published authors. 